\title{
Review
}

Cerebrovascular

Diseases
Cerebrovasc Dis 2014;38:313-323

DOI: $10.1159 / 000365864$
Received: April 9, 2014

Accepted: July 2, 2014

Published online: November 21, 2014

\section{Surgical Aspects of Decompression Craniectomy in Malignant Stroke: Review}

\author{
Klaus Zweckberger $^{\text {a }} \quad$ Eric Juettler ${ }^{c}$ Julian Bösel ${ }^{b} \quad$ W. Andreas Unterberg ${ }^{a}$ \\ Departments of a Neurosurgery and ${ }^{\mathrm{b}}$ Neurology, University Heidelberg, Heidelberg, and ${ }^{\mathrm{c}}$ Department of Neurology, \\ University UIm, UIm, Germany
}

\section{Key Words}

Middle cerebral artery infarction · Surgical decompression . Hemicraniectomy $\cdot$ Indications for surgery

\begin{abstract}
Background: Space-occupying malignant stroke of the middle cerebral artery (MCA) is associated with a high mortality rate of up to $80 \%$ under conservative treatment. Although there is convincing evidence that decompression craniectomy can significantly reduce mortality rate and improve neurological outcome in young patients ( $<60$ years), many surgeons are still hesitant to recommend hemicraniectomy for stroke patients. Summary: This review addresses some major issues that appear to be an obstacle to decompression craniectomy, in particular, indicating surgery for patients $>60$ years or with infarcts of the dominant hemisphere. Furthermore, it emphasizes technical issues such as timing and size of the craniectomy, additional temporal lobectomy, and resection of the temporal muscle, as well as duraplasty and cranioplasty. According to the current literature, decompression craniectomy in older patients can increase survival without most severe disabilities, although, most survivors need assistance in most bodily needs. Involvement of the dominant hemisphere results in aphasia that might partly recover in younger patients, although, considering the neuropsychological deficits caused by infarctions of the non-
\end{abstract}

dominant hemisphere, involvement of the dominant hemisphere does not pose as a contraindication for decompression craniectomy. Furthermore, there is convincing evidence that surgery should be performed within $48 \mathrm{~h}$ after the onset of symptoms and the size of the craniectomy should be at least $12 \mathrm{~cm}$ as a minimum. An additional lobectomy or the resection of the temporal muscle, however, can only be part of individual treatment options. Conceding the weak evidence, it is recommended to close the dura by some form of a duraplasty avoiding cerebrospinal fluid leakages or scarring between the cortex and the scalp leading to injuries during reimplantation of the bone-flap. Complications associated with decompression surgery (hemorrhages, infections, 'sinking skin-flap syndrome', cerebrospinal fluid leakages, hydrocephalus, seizures), with the infarction itself, or with those that occur during the ICU course (cardiac and pulmonary complications) appear acceptable and are mostly treatable, especially considering the fatal course of conservative treatment. Key Message: This review summarizes the current state of the literature about decompression craniectomy of patients with malignant stroke addressing, in particular, critical surgical issues, and thus, help surgeons to make decisions confidently for/or against performing surgery.

(c) 2014 S. Karger AG, Basel

None of the authors has any conflict of interest or received financial support for this review.

\section{KARGER}

E-Mail karger@karger.com

www.karger.com/ced
(C) 2014 S. Karger AG, Basel

$1015-9770 / 14 / 0385-0313 \$ 39.50 / 0$
Dr. med. Klaus Zweckberger, MD, PhD

Department of Neurosurgery, University Hospital Heidelberg

Im Neuenheimer Feld 400

DE-69120 Heidelberg (Germany)

E-Mail klaus.zweckberger@web.de and klaus.zweckberger@med.uni-heidelberg.de 

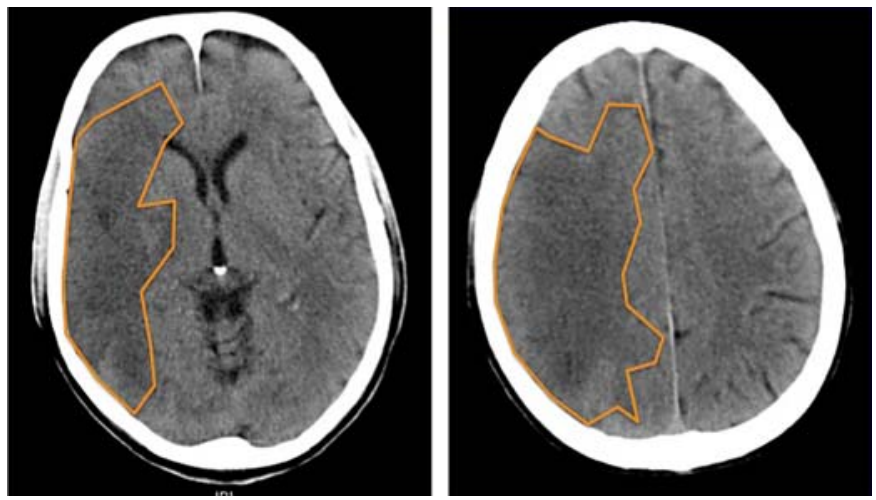

Fig. 1. CT images of a 46-year-old patient with a space-occupying 'malignant' MCA infarction of the right hemisphere.

\section{Introduction}

Space-occupying infarctions of the middle cerebral artery (MCA) that may also include parts of the anterior (anterior cerebral artery, ACA) or posterior (posterior cerebral artery, PCA) territories constitute up to $10 \%$ of all supra-tentorial strokes $[1,2]$ (fig. 1). Characteristically, this type of infarction is associated with substantial brain edema, brain shift, increased intracranial pressure (ICP), and thus, uncal or trans-tentorial herniation, finally leading to brain death [3-6]. Clinically, patients present symptoms including contralateral hemiplegia or hemiparesis, aphasia if the dominant hemisphere is affected, and head or eye deviation. Because of the development of excessive brain edema, patients deteriorate uniformly and rapidly within 2-5 days after onset of symptoms. Under standard conservative care, these space-occupying MCA infarctions are affiliated with a fatality rate of up to $80 \%$ $[2,3,6]$. Reflecting the fatal course of this type of infarction, the expression malignant stroke was coined [3]. While this type of infarction was considered not treatable but fatal for a long time, especially since the increasing number of patients more from the younger generation, further treatment options became necessary [3]. Decompression craniectomy is not a new idea and has been used as an individual treatment option in order to control extending brain edema and to improve cerebral perfusion pressure for many decades. Since the publication of prospective randomized trials (DECIMAL, HAMLET, DESTINY I, Zhao et al., DESTINY II, HeADDFIRST) [7$13,26]$ showing a significant reduction in mortality rate and an improvement in neurological outcome, evidence for decompression craniectomy is growing. While it has consequently been implemented in the treatment of ma- lignant stroke, many surgeons remain skeptical and often have several caveats indicating hemicraniectomy. In particular, and with respect to the neurological outcome, the age of the patient and involvement of the dominant hemisphere are critical issues. Furthermore, technical aspects such as the size of the craniectomy, performing an additional temporal lobectomy, or the use of different materials for the dura-plasty, as well as the timing of surgery itself, are broadly underestimated in many trials, and therefore, are set in the focus of this review, conceding that the body of evidence is weak in many critical issues.

\section{Conservative Treatment}

The primary aim of any treatment approach for acute space-occupying brain infarction constitutes the control over the excessive brain edema development and the improvement of cerebral perfusion pressure and oxygen tissue saturation. Most patients are stuporous or comatose and in order to prevent them from aspiration, intubation, sedation, and mechanical ventilation are frequently necessary [14]. While many physicians on neuro intensive care units support ICP monitoring in unconscious patients, it is still debatable whether an ICP-targeted therapy might improve outcome of stroke patients. Poca et al. could not prove any advantage of ICP monitoring in comparison to clinical examinations and CT scanning [15]. Medical treatment of cerebral edema mainly consists of osmotherapy with mannitol or hypertonic saline, sedation, and eventually hyperventilation, buffers, or hypothermia [16, 17]. It should, however, be considered that none of these treatment options have been investigated in randomized clinical trials, so far [18]. Direct comparison of equiosmolar doses of mannitol (20\%) and hypertonic saline (23.4\%) has shown comparable effects on cerebral blood flow (CBF) in positron emission tomography (PET), but unchanged cerebral blood volumes (CBV) [19]. Of note, this work did not confirm alleged fears of osmotic agents actually residing in the infarcted tissue and subsequently promoting edema development and mid-line shift. A recently published meta-analysis considering 112 patients of 5 trials describe an advantage for hypertonic therapy in comparison to mannitol in order to control elevated ICP with a mean difference of $2 \mathrm{~mm}$ $\mathrm{Hg}$ [20]. In general, more neurointensivists favor the use of hypertonic saline for a reduced frequency of rebound edema, less side effects, desired volume expansion, and a longer-lasting duration of therapeutic effects [21]. Furthermore, therapeutic hypothermia $\left(33^{\circ}\right)$ offers a neuro- 
protective and anti-edematous therapy with proven efficiency in animal models and clinical trials after global ischemia, albeit proof of efficiency is still lacking for focal cerebral ischemia [22-24]. Currently, the DEPTH-SOS trial investigates the combined effect of decompression craniectomy and therapeutic hypothermia on the mortality rate and on the neurological outcome of patients with space-occupying stroke [25].

Despite optimizing conservative treatment modalities, the mortality rate has remained high $(>70 \%)$, displayed in the control arms of the current prospective randomized trials [7-10], and did not show significant differences compared to palliatively treated patients (80\%) [3].

\section{Surgical Treatment}

\section{Controlled Randomized Clinical Trials}

Based on the promising results of experimental research and nonrandomized clinical studies, meanwhile eight randomized controlled trials have been initiated. While the Turkish DEMITUR trial is completed, but still unpublished, and the HeMMI study (Hemicraniectomy for middle cerebral artery infarction) is still recruiting, six trials have been finalized (DECIMAL, HAMLET, DESTINY I, Zhao et al., DESTINY II, HeADDFIRST). In addition, a prospective pooled data analysis of DECIMAL, HAMLET, and DESTINY I has been performed including patients treated within 48 hours. With the exception of the HeADDFIRST trial, all these studies show a significant reduction in the mortality rate and improved outcome [7-10, 13].

The German DESTINY I study (Decompressive Surgery for the Treatment of Malignant Infarction of the Middle Cerebral Artery) included 32 patients from 18 to 60 years and an NIHSS score of $>18$ for the nondominant and $>20$ for the dominant hemisphere. On CT scans, twothirds of the MCA territory and at least parts of the basal ganglia had to be involved. Patients were randomized within $12-36 \mathrm{~h}$ following the onset of symptoms. Patients who received surgical decompression craniectomy showed a significantly reduced 1 -year mortality rate $(18 \%$ vs. $53 \% ; \mathrm{p}=0.03$ ) and an improved neurological outcome in the Modified Rankin Score (mRS) after 180 days and after 1 year, respectively (mRS 2-3 (180 days): 47\% vs. $27 \%$; mRS $2-3$ (1 year): $48 \%$ vs. $27 \%$ ) [8].

The French DECIMAL study (Decompression Craniectomy in Malignant Middle Cerebral Artery Infarction) enrolled 38 patients younger than 55 years and with an NIHSS score $>15$. Patients were randomized to decom-

Surgical Aspects of Decompression

Craniectomy in Stroke pression surgery versus best medical treatment within 30 hours following stroke. The 1-year mortality rate was significantly reduced in surgically treated patients ( $25 \%$ vs. $78 \% ; \mathrm{p}=0.0001$ ) and modified Rankin Scores showed a nonsignificant trend of neurological improvement ( $\mathrm{mRS}$ $\leq 3$ ( 6 months): $25 \%$ vs. $5.6 \%$; $p=0.18$ and $m R S \leq 3$ ( 1 year): $50 \%$ vs. $22.2 \%$; $=0.1)$. A comparison of outcomes according to non-dichotomized scores on the mRS differed significantly between the two groups at 6 and 12 months follow-up in favor for craniectomy $(\mathrm{p}=0.011$ and $\mathrm{p}=$ 0.0024 , respectively) [9].

The Dutch HAMLET study (Hemicraniectomy after Middle Cerebral Artery Infarction with Life-threatening Edema Trial) recruited 64 patients between 18 and 60 years with an infarction including at least two-thirds of the MCA territory associated with space-occupying edema on CT scans. Randomization was performed within 96 hours after onset of symptoms, and thence, significantly later compared to the DECIMAL and DESTINY trials. As a secondary endpoint, mortality rate in the surgical group was not so significant after 1 year $(22 \%$ vs. $59 \% ; \mathrm{p}=0.002)$. Neurological outcome, however, could not be improved (mRS 4-6: 75\% vs. 75\%; mRS 5-6: 41\% vs. 59\%) after 1 year. A subgroup of patients who was operated within 48 hours, however, showed significant better neurological outcomes [7].

In the pooled analysis of the DESTINY I, DECIMAL, and HAMLET trials, 93 patients with an age ranging between 18 and 60 years, an NIHSS score $>15$, and an infarction size greater than two-thirds of the MCA territory on CT scans or an infarct volume of at least $145 \mathrm{~cm}^{3}$ on DWI scans, were randomized to decompression surgery or best medical treatment within $48 \mathrm{~h}$ after the onset of symptoms. Decompression craniectomy resulted in an absolute risk reduction of $50 \%$ for mortality, of $42 \%$ for $\mathrm{mRS} \geq 4$, and of $16 \%$ for $\mathrm{mRS} \geq 3$ after 12 months. Seventy five percent of surgically treated patients achieved an mRS of $0-4$ (vs. $24 \%$ in the conservative treatment arm, $\mathrm{p}=0.0001$ ). $43 \%$ achieved an $\mathrm{mRS}$ of $0-3$ (vs. $23 \%$, $\mathrm{p}=0.014)$, respectively [10].

Despite this convincing body of evidence derived from the European studies, these results could not be confirmed in the currently published HeADDFIRST trial. Among the 4.909 patients screened from 2000 to 2002, finally only 24 patients were randomized in a medical treatment $(\mathrm{n}=10)$ or a medical treatment plus decompression surgery $(\mathrm{n}=14)$ group. Acute mortality (21 days after stroke) was reduced in surgically decompressed patients by $19 \%$ ( $21 \%$ medical treatment plus surgery vs. $40 \%$ medical treatment, $\mathrm{p}=0.39$ ), but this difference has 
already been redeemed within 180 days ( $36 \%$ vs. $40 \%$ ). Furthermore, there was no significant effect on neurological outcome 180 days after stroke. Authors explained these differences, as well as the small fatality rate in conservatively treated patients, compared to the European trials mainly based on the randomization process and the study protocol, having 'stricter' inclusion criteria identifying patients on risk for excessive edema, incorporating older patients (up to 75 years), and having a wider time window for decompression surgery (until $96 \mathrm{~h}$ ). These conclusions, however, contradict the discoveries derived from the HAMLET and other European trials [7-10, 13].

Despite the convincing and promising results from the European trials, and partly since the contradicting results from the HeADDFIRST trial [26], many surgeons are still hesitant to indicate decompression craniectomy apparently under the conception that reducing the mortality rate may result in an increased amount of severely disabled and dependent patients in which quality of life seems unacceptably poor. The remainder of this review therefore focuses on some of the most important obstacles that come in the way of performing a decompression surgery for patients with malignant stroke.

\section{Timing for Decompression Surgery}

Timing of surgery seems to be an important factor for mortality and neurological outcome. There are two studies explicitly emphasizing this issue. Already in 1998, Schwab et al. examined the effect of early ( $21 \mathrm{~h}$ ) versus late decompression craniectomy $(39 \mathrm{~h})$ in 63 patients showing a decreased mortality rate (16\% vs. $34 \%)$ and a reduced rate of herniation ( $13 \%$ vs. $75 \%$ ) in early treated patients, as well as a shorter time on the ICU (7.4 vs. 13.3 days) [27]. In a retrospective trial on 21 patients, Wang et al. showed the effect of an early decompression craniectomy versus late decompression ( $<24 \mathrm{~h}$ vs. $<24 \mathrm{~h}$ after onset of symptoms). The mortality rate was equal, but severe disability could be reduced in early treated patients [28]. Both in the DECIMAL and DESTINY trials, decompression craniectomy was performed within 43 or $36 \mathrm{~h}$ after the onset of symptoms, respectively $[8,9]$, resulting in reduced early and long-term mortality rates and improved neurological outcomes. The HAMLET trial, allowing decompression surgery up to $96 \mathrm{~h}$, did show a significant reduction in the mortality rate after one year or improved neurological outcome, at least not in patients treated after $51 \mathrm{~h}$ of onset of symptoms [7]. Several retrospective studies have included patients who have already shown uncal hernation and one dilated pupil. Maramottom et al. described in a nonrandomized study on 24 pa- tients ( 14 were treated by decompression surgery) a mortality rate of $57 \%$ in surgically treated patients, and $0 \%$ in patients who received conservative treatment, showing that late decompression surgery after onset of herniation is not beneficial [29]. Similar effects were seen in the prospective study of Malm et al. [30]. Out of 30 enrolled patients 14 showed signs of herniation (dilated pupil), and the time point of surgery varied broadly from 13 to $235 \mathrm{~h}$ after stroke [25]. Likewise, these results are supported by a prospective study of Kilincer et al. identifying sings of herniation as a prognostic factor for poor outcome [31], and by several retrospective trials [32, 33, 34]. Furthermore, in the studies of Skoglund et al. $(\mathrm{n}=18)$ and Chung et al. $(\mathrm{n}=24) 67$ and $100 \%$ of patients, respectively, showed signs of herniation, leading to worse neurological outcomes compared to the results of the DECIMAL and DESTINY trials $[8,9,35,36]$.

On the other hand, Cho et al. described in a small cohort of 12 patients a positive effect of ultra-early decompression craniectomy performed within $6 \mathrm{~h}$ after the onset of symptoms. The acute mortality rate was $8.3 \%$, compared to $36.7 \%$ for delayed decompression surgery, and $80 \%$ for conservative treatment [37].

While there are reservations that very early decompression surgery might potentially be unnecessary, it is clear that decompression surgery beyond $48 \mathrm{~h}$ after the onset of symptoms is less effective, or may even be ineffective in order to reduce mortality and to improve neurological outcome, especially when waiting until signs of herniation are present.

\section{Size of the (Hemi-)Craniectomy}

As a matter of principle, hemicraniectomy and durotomy are performed in order to allow the infarcted hemisphere to swell and to avoid further compression and damage of adjacent cortical structures and to prevent herniation. Besides the size of the craniectomy itself, osteoclastic temporal enlargement is particularly important to decompress the middle cranial fossa. A suboptimal size smaller than $12 \mathrm{~cm}$ in diameter has been described to cause additional cortical lesions leading to a decreased survival rate (55\% vs. $80 \%$ ) [38] (fig. 2). Most studies postulate a diameter of at least $12 \mathrm{~cm}$ as an absolute minimum [27, 30, 39-41]. Likewise in the prospective trials (DESTINY I, DECIMAL, HAMLET) and their pooled analysis, a diameter of at least $12 \mathrm{~cm}$ was claimed [7-10] (fig. 3).

Some smaller studies, however, describe even larger sizes of more than $14 \mathrm{~cm}$ in diameter $[28,42,43]$ or including the superior sagittal sinus [44]. Walz et al. de- 


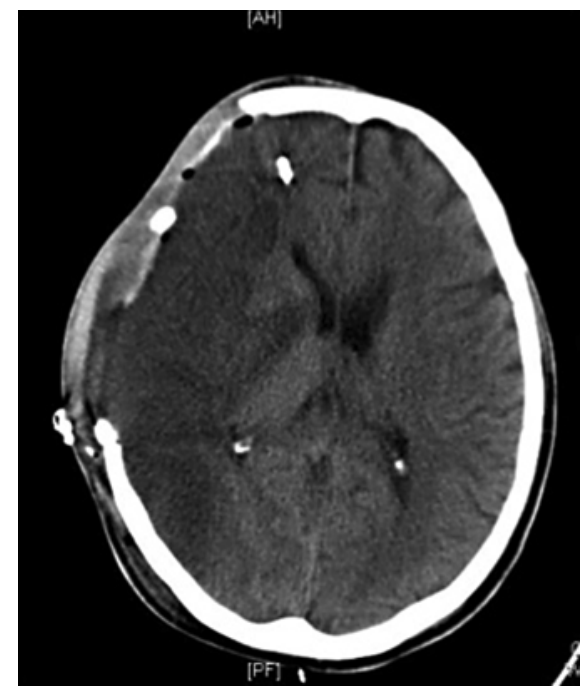

Fig. 2. CT scanning of a patient with a malignant MCA infarction of the right hemisphere after decompression craniectomy. The size of the craniectomy is too small, and thus the infarction is not decompression sufficiently.
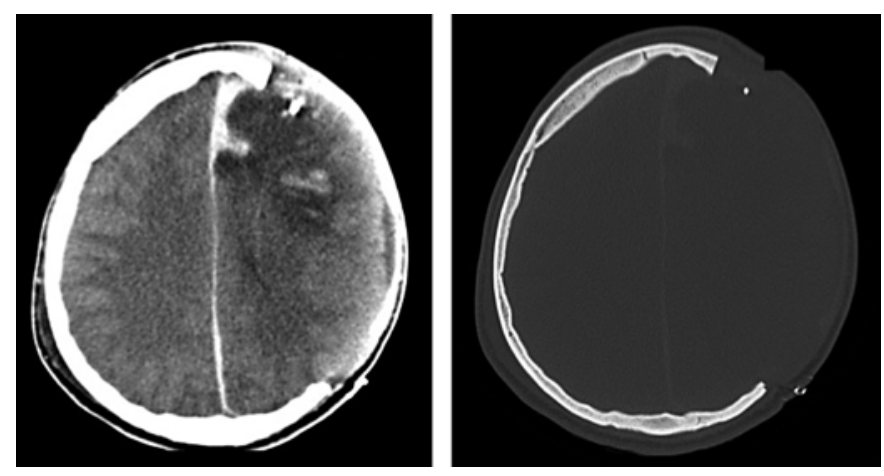

Fig. 3. CT scanning of a patient following decompression craniectomy showing a large and sufficient size of the hemicraniectomy.

scribed an acute mortality rate in the first week of only $11 \% .91 .6 \%$ of survivors showed moderate or slight disabilities and only $8.3 \%$ severe dependency after 14 months [42]. Furthermore, Park et al. emphasize the effect of an additional resection of the temporal muscle optimizing the decompressive effect of the craniectomy $(>12 \mathrm{~cm})$. $20 \%$ of the operated patients died. Although $60 \%$ of the survivors showed an $\mathrm{mRS}<4,20 \%$ remained severely disabled and dependent ( $\mathrm{mRS} \geq 4$ ) in a 6 months follow-up assessment [45].

In a recently published study, Chung et al. could demonstrate that a maximal size of decompression craniectomy $\left(>14-16 \mathrm{~cm}\right.$ or $\left.>399 \mathrm{~cm}^{2}\right)$ versus a large size $(>12$

Surgical Aspects of Decompression

Craniectomy in Stroke $\mathrm{cm}$ or $308 \mathrm{~cm}^{2}$ ) increases the ratio of a favorable outcome 3 months after stroke $(\mathrm{mRS} \leq 3)$ and decreases the ratio of poor outcomes or deceased patients [36].

While there is growing evidence that large craniectomies ( $>14 \mathrm{~cm}$ in diameter) seem to predict favorable outcome, there is no evidence derived from clinical randomized prospective trials that an enlargement of the craniectomy including the superior sagittal sinus or of the resection of the temporal muscle are beneficial. Giving any recommendations these aspects should first be examined in clinical randomized prospective trials.

\section{Temporal Lobectomy}

Some studies describe the attempt to pull up the decompressive effect of the craniectomy and to prevent further brainstem compression by performing an additional temporal lobectomy. This issue, however, is not examined in clinical randomized trials. Greenwood, one of the first who has described surgery for brain infarction, reported successful outcomes in 6 out of 9 patients following craniectomy and resection of infarcted brain tissue [46]. Though there are some retrospective studies and case reports acknowledging successful anterior lobectomies [47], it has not been consistently shown yet that this procedure has beneficial effects on the mortality rate or on neurological outcome compared to patients with an adequately sized craniectomy alone [32, 42, 43]. In none of the prospective controlled randomized multi-center trials lobectomies were conducted (DECIMAL, DESTINY I, HAMLET) [7-9].

On the other hand, there are certain concerns regarding the risk of hemorrhages in resecting fragile infarcted brain tissue, particularly if the patients have been treated with aspirin, which is often part of the initial stroke management.

Based on the current literature and owing to the lack of evidence, it remains unclear if additional lobectomy might be beneficial. Giving any recommendation this issue should first be addressed in clinical randomized prospective trials.

\section{Duraplasty}

In order to achieve a maximal decompressive effect of the craniectomy, the opening of the dura is mandatory. To reduce the risk of cerebral spinal fluid (CSF) leakages, most neurosurgeons might advocate some form of dura closure. Considering the expansion of the underlying brain tissue, the use of a duraplasty seems to be necessary. Another objection to leaving the dura open are potential problems that might occur during subsequent cranioplas- 
ty. Adhesions and scars formed between the cortex, the scalp, or the temporal muscle could induce injuries of the cortex and bleedings during the preparation for reinsertion of the bone flap. The literature suggests that different materials may be used for duraplasty, including artificial ones such as bio-design grafts or Gore-Tex ${ }^{\circledR}$, as well as autologous ones, such as fascia lata or galea periost.

As an alternative to the 'classical duraplasties' Gueresir et al. described on more than 300 patients a 'rapid closer technique' using haemostyptic materials ('Surgicel') in order to create a scarring layer between the cortex and the scalp not increasing the complication rate, especially for CSF leakages $(<1 \%)$ or abscesses $(<2.6 \%)$ [48]. Alternatively, the formation of such a layer may be done by the use of silicone or poly-tetra-fluoro-ethylene sheets [49, $50]$.

Nevertheless, no differences on complication rates or on the clinical outcome could be reported so far, conceding that there are no studies available comparing different materials in particular.

\section{Cranioplasty - Reinsertion of the Bone Flap}

Timing for reinsertion of the bone flap varies considerably between 6 weeks and 6 months. There is no study however, that examines the effect of timing on the clinical outcome of patients. In accordance with the available literature, timing does not influence complications, but there might be an advantage for early reinsertion ( $<3$ months), especially with respect to the risk for infections [51-56]. Recently published studies reported an approximate overall complication rate of up to $35 \%[51,52]$. $26 \%$ of patients had minor complications including seizures $(15.6 \%)$ or subgaleal fluid collections $(3.1 \%)$, and superficial wound infections (3.1\%), whereas major complications (10.4\%) encompass hydrocephalus (3.1\%), transient neurological deficits (3.1\%), and osteomyelitis (2.1\%) [51]. Wachter et al. reported about a complication rate of $30 \%$ with $17.4 \%$ aseptic bone necrosis, $5 \%$ wound infections, $2.5 \%$ hematoma, $2.7 \%$ hygroma, and $1.7 \%$ unsatisfying cosmetic results [53]. In a large series of 239 patients, Walcott et al. described an overall complication rate of $24 \%$. They could not find predicting factors such as age, location of the cranioplasty, presence of an intracranial device (like an ICP probe), bone flap preservation method, cranioplasty material, and time interval $>90$ days between initial craniectomy and cranioplasty [57]. On the other hand, re-operation was clearly identified as an independent risk factor [57]. Considering the dire outcome of conservative treatment affiliated with a fatality rate of $70-80 \%$, these mostly minor complications appear ac- ceptable, are usually easy to treat, and should not constitute an argument not to perform decompression craniectomy.

\section{Age Limit}

The pooled analysis of the three prospective trials (DECIMAL, DESTINY I, HAMLET) showed a significant reduction in mortality rate by almost $50 \%$ and a significant improvement in the clinical outcome in patients younger than 60 years. Furthermore, in a retrospective database analysis of 188 patients Uhl et al. showed encouraging effects for decompression craniectomy in space-occupying MCA infarctions in patients $<50$ years, but the results for older patients remained questionable [58]. Hence, it was doubtful for a long time if patients older than 60 years might also benefit from decompression craniectomy [7-10, 58].

Regardless of other factors, age is an independent predictor for poor outcome. In 2001, Holtkamp et al. assessed the effect of decompression craniectomy on the outcome of patients between 55 and 75 years of age. Even though they could show a reduction in mortality in the surgical group (33\% vs. $75 \%$ ), the neurological outcome was poor [39]. Their results were supported by several clinical series $[31,33,34,41-43,58,59]$. In a review Arac et al. reported about 273 patients from 19 studies showing a mortality rate of $51 \%$ in the sub-group analysis of patients older than 60 years versus $21 \%$ in younger patients, associated with a significantly larger number of patients with poor neurological outcomes in the subgroup $>60$ years $(81.8 \%$ vs. 33.1\%) [60]. Although there are several studies indicating less beneficial effects for older patients, there was a dearth of controlled prospective randomized trials. A problem of most of these studies is that older patients were often operated much later and did often not receive maximal conservative treatment. The recently published prospective randomized controlled clinical multi-center DESTINY II trial investigated for the first time the effect of decompression craniectomy on older patients in an adequately powered randomized study $[12,61]$. DESTINY II enrolled 112 patients older than 60 years (median age 70 years, range 61-82 years) within $48 \mathrm{~h}$ after onset of symptoms [61]. At the primary endpoint after 6 months, mortality rate was reduced in the hemicraniectomy group (33\% vs. $70 \%)$. No patients, however, survived with an mRS $0-2$, and only $7 \%$ in the surgical arm reached an mRS of 3 compared with $3 \%$ in the conservative arm. The rates for mRS of 4 were 32 and 15, respectively. Modified RS of 5 was more frequent in surgically treated patients (28\% vs. $13 \%)$. At the secondary endpoint after 12 months hemi- 
craniectomy still supports survival, although the total number of deaths has increased in both groups ( $43 \%$ vs. $76 \%$, respectively). Overall, $38 \%$ of the surgically treated patients, compared with $16 \%$ of the conservatively treated ones, achieved an mRS $\leq 4$. In conclusion, early hemicraniectomy increases the probability of survival, but most survivors will have substantial disabilities $[12,61]$. Furthermore, in a recently published prospective randomized trial Zhao et al. reported slightly better results in a subgroup analysis of patients older than 60 years who received decompression craniectomy showing a reduced mortality rate (19\% vs. $69 \%)$ and less patients with poor neurological outcomes (mRS 4-5) [11].

In accordance with the available literature, there is convincing evidence that decompression craniectomy improves the chance for survival, also in patients older than 60 years. An overwhelming number of patients, however, remain dependent (mRS 4 or 5), and only few achieve an mRS of 3. Especially the number of patients remaining in complete dependency ( $\mathrm{mRS}$ of 5 ) is considerably high (20\%) compared with younger patients (4\%) $[10,12,61]$. There is still a controversial discussion about the acceptability of such a neurological outcome when the alternative is death. Although, in many studies an age of 60 years distinguishes between old and young patients with malignant MCA infarction, the physiological constitution of the individual patient, and his/her will to accept severe handicaps instead of being dead, especially between 60 and 70 years, varies considerably. Therefore, it is problematic to state a cut-off age of 60 years. Instead, it is recommendable to discuss potential chances of surgery, but also associated risks for poor outcome with the patients' relatives and to explore the potential attitude of the patient towards a life-saving procedure potentially associated with severe disabilities. Nevertheless, many people have reservations about a life-saving surgical procedure for the price of being highly disabled and dependent. In a population-based epidemiological study, 312 adults were surveyed presenting a scenario of space-occupying MCA infarction. Only $2 \%$ favored surgical intervention, $58 \%$ were uncertain, and $39 \%$ were opposed to surgery. The number of individuals opting for surgery increased in better scenarios. If a poor outcome (mRS 4 or 5 ) was anticipated, only 1 or $6 \%$ favored surgery, respectively [62].

\section{Infarction of the Dominant Hemisphere}

Especially with respect to the patients' neurological outcome it is still under debate whether decompression craniectomy of the dominant hemisphere is recommendable. The impact of the dominant hemisphere is not suf-

Surgical Aspects of Decompression

Craniectomy in Stroke ficiently considered in available studies of malignant MCA infarction, and moreover, most studies include a mixture of dominant and nondominant stroke patients, or even mention only the number of patients with aphasia. The present overview shows the rate of dominanthemispheric infarction represented in the literature: Holtkamp et al. (both sides without differentiation) [39], Skoglund et al. (17\%) [35], Foerch et al. (19\%) [33], Maramattom et al. (22\%) [29], Rabinstein et al. (24\%) [59], Robertson et al. (25\%) [32], Harscher et al. (27\%) [41], Yao et al. (32\%) [63], Weil et al. (36\%) [64], Chen et al. (37\%) [34], Jüttler et al. (37\%) [12], Jüttler et al. (38\%) [8], Hofmeijer et al. (38\% aphasia) [7], Zhao et al. (38\%) [11], Wang et al. (38\%) [28], Koh et al. (43\%) [65], Walz et al. (44\%) [42], Malm et al. (47\%) [30], and Kiphuth et al. (57\%) [66], Vahedi et al. (60\% aphasia in the conservative arm, and $55 \%$ in the surgical arm, as an outcome parameter) [10], Vahedi et al. (60\% survivors with aphasia after 1 year) [9]. Only few studies found a difference in outcome after malignant MCA infarction that is influenced by the side of the lesion. The reason why most studies do not find any differences in outcome according to the hemisphere is the fact that most studies use the mRS as the main outcome parameter, where aphasia is not represented as a major parameter determining outcome. Aphasia is, however, the main, or often the only argument, why decrompressive surgery is more reluctantly addressed in malignant stroke of the dominant hemisphere. Patients nevertheless have a chance for language recovery. Young age and early decompression surgery in patients with malignant dominant stroke have been identified as predictors for language recovery, conceding that this might take several months or even years and might be incomplete [67]. While the recovery of language is modulated by several factors including type, intensity, and timing of the intervention or the severity of the initial presentation, the anatomical regions for recovery are less clear [68-71]. A growing body of evidence originating from transcranial magnet stimulation studies (TMS) shows functional reorganization of language in aphasic patients [72]. While it is broadly assumed that shifting of functional language regions to the nondominant hemisphere might establish the foundation for recovery, current fMRI studies, however, demonstrate that patients with normal or close-tonormal language functions at least 1 year after stroke show a return to typical fMRI activation patterns when compared to the results of fMRI data obtained in previous studies that included healthy controls and comparable language tasks $[73,74]$. Reorganization of language functions by shifting to the right hemispheric brain regions 
was shown to be a less effective mode of language function recovery [73-75].

Though it might be problematic to indicate decompression craniectomy in older patients considering aphasia, involvement of the dominant hemisphere does not pose a contraindication for younger patients and should be performed as early as possible $[67,74]$. Nevertheless, in two-thirds of involved patients, aphasia remained unchanged 12 months later and neuropsychological testing has revealed that $62 \%$ of the aphasic patients fulfilled the DSM-III-R criteria for depression 12 months after stroke. Furthermore, the prevalence of major depression increased within the first year from $11 \%$ to $33 \%$ [76]. These results emphasize the necessity for long-lasting neurological rehabilitation and neuropsychological care for aphasic patients.

Nevertheless, it must be pointed out that the severe neuropsychological deficits of nondominant hemispheric malignant infarcts might probably be least equally disabling as aphasia and depression is just as frequent in nondominant hemispheric infarcts. Except for studies on communication, ability studies on the quality of life or activities of daily living found no differences in any other domain between patients with malignant infarcts of the dominant and those of the nondominant hemisphere [12, $42,77]$.

\section{Complications}

Complications following decompression craniectomy after malignant stroke must be differentiated in acute complications caused by the surgical procedure itself, complications occurring in the ICU, and delayed ones, for example, the development of a hydrocephalus or any associated ones with the cranioplasty. In fact, this aspect of decompression surgery for stroke has never been systematically assessed and all of the following are based on case series, case reports, or reports as part of adverse events listed in trials not focusing on complications in the first place. Although decompression craniectomy is an easy and safe procedure, the rare occurrence of severe complications might adversely affect the outcome of patients.

Complications following decompression craniectomy include hematomas, meningitis, and wound infections. Epidural hemorrhages (EDH) or parenchymal lesions were rarely reported and occurred only in exceptional cases, although, the number of cerebral spinal fluid (CSF) leakages remained indistinct $[27,38,44,77,78]$. The number of seizures has varied significantly and ranged from $11 \%$ up to $66 \%$, although, it was not clearly shown whether their occurrence was a consequence of the surgical procedure or a more general phenomenon following stroke $[44,79]$. In most studies, infection rates were not explicitly outlined. Investigations on trauma patients revealed an overall infection rate of about $11 \%$, and a reabsorption rate of the bone flap of $7 \%$ following decompression craniectomy [80].

Notably, during the ICU treatment, cardiac and pulmonary complications may occur. Durga et al. have described a mortality rate of $36 \%$ as a consequence of cardiac disturbances [81]. Pneumonia, sepsis, and pulmonary embolism were frequent and often resulted in fatal complications during the ICU treatment [82].

The so-called 'sinking skin flap syndrome' might result in a paradoxical herniation, headaches, seizures, and focal neurological deficits [83-85]. In the DECIMAL trial, the authors have addressed this issue and suggested that the 'sinking skin flap syndrome' might be associated with smaller sizes of the craniectomy [9]. Furthermore, $62 \%$ of patients developed an extra-axial fluid collection, even though half of them had no ventricular drainage [86]. Delayed hydrocephalus, however, is a common phenomenon following stroke and has occurred in up to 30 or $47.8 \%$ of patients, respectively $[87,88]$. The presence of pre-, or post-cranioplasty hydrocephalus might have negative effects on the neurological outcome and could be associated with a craniectomy limit $<2.5 \mathrm{~cm}$ from the midline [89]. Early reinsertion of the bone flap might address this problem and help to prevent development of excessive axial fluid collections [86, 90].

Although the overall complication rate seems to be high, severe surgery-related complications are rare and often do not require revision. If they do, they are mostly well treatable (e.g., shunts for hydrocephalus), and thus appear acceptable, especially considering the fatal course associated with conservative treatment $(80 \%$ mortality rate).

\section{Summary and Conclusion}

With reference to the available literature, there is convincing evidence that decompression craniectomy can significantly reduce the mortality rate and improve the neurological outcome in patients with malignant stroke. This applies especially to patients younger than 60 years who received an early ( $<48 \mathrm{~h}$ after onset of symptoms) and large (at least 12-14 cm) decompression craniectomy, whereas older patients have a less favorable outcome, although in older patients mortality and most se- 
vere disability are also significantly reduced by early hemicraniectomy, conceding that many patients remain highly disabled and dependent. While it was clearly shown that an insufficient size of the craniectomy $(<12$ $\mathrm{cm}$ ) has a negative impact on the outcome, there is no evidence that additional lobectomy or resection of the temporal muscle might influence the mortality rate or the neurological outcome. Extra-large $(>14 \mathrm{~cm})$ and very early $(<48 \mathrm{~h})$ craniectomies might further improve the mortality rate and outcome, but this needs to be demonstrated in future studies. Considering possible capabilities for recovery from aphasia, especially in younger patients, the involvement of the dominant hemisphere does not constitute a contraindication for surgery. Despite considerable rates of physical disability and depression, the vast majority of patients are satisfied with their quality of life after treatment and do not regret having undergone a surgery [91].

\section{References}

$>1$ Ropper AH, Shafran B: Brain edema after $>1$ stroke. Clinical syndrome and intracranial pressure. Arch Neurol 1984;41:26-29.

2 Silver FL, Norris JW, Lewis AJ, et al: Early mortality following stroke: a prospective review. Stroke 1984;15:492-496.

3 Hacke W, Schwab S, Horn M, et al: 'Malignant' middle cerebral artery infarction: clinical course and prognostic signs. Arch Neurol 1996;53:309-315.

-4 Heinsius T, Bogousslavsky J, Van Melle G: Large infarcts in the middle cerebral artery territory. Etiology and outcome patterns. Neurology 1998;50:341-350.

5 Sakai K, Iwahashi K, Terada K, et al: Outcome after external decompression for massive cerebral infarction. Neurol Med Chir 1998;38: 131-135.

6 Shaw CM, Alvord EC Jr, Berry RG: Swelling of the brain following ischemic infarction with arterial occlusion. Arch Neurol 1959;1: 161-177.

7 Hofmeijer J, Kappelle LJ, Algra A, et al: Surgical decompression for space-occupying cerebral infarction (the Hemicraniectomy After Middle Cerebral Artery infarction with Lifethreatening Edema Trial HAMLET): a multicenter, open, randomised trial. Lancet Neurol 2009;8:326-333.

8 Jüttler E, Schwab S, Schmiedek P, et al: Decompressive Surgery for the Treatment of Malignant Infarction of the Middle Cerebral Artery (DESTINY): a randomized, controlled trial. Stroke 2007;38:2518-2525.

$\checkmark 9$ Vahedi K, Vicaut E, Mateo J, et al: Sequentialdesign, multicenter, randomized, controlled trial of early decompressive craniectomy in malignant middle cerebral artery infarction (DECIMAL Trial). Stroke 2007;38:2506-2517.

10 Vahedi K, Hofmeijer J, Juettler E, et al: Early decompressive surgery in malignant infarction of the middle cerebral artery: a pooled analysis of three randomised controlled trials. Lancet Neurol 2007;6:215-222.

11 Zhao J, Su YY, Zhang Y, et al: Decompressive hemicraniectomy in malignant middle cerebral artery infarct: a randomized controlled trial enrolling patients up to 80 years old. Neurocrit Care 2012;17:161-171.
Jüttler E, Unterberg A, Woitzik J, et al; DESTINY II Investigators: Hemicraniectomy in older patients with extensive middle-cerebral-artery stroke. N Engl J Med 2014;370: 1091-1100.

13 Frank JI, Schumm LP, Wroblewski K, et al; HeADDFIRST Trialists: Hemicraniectomy and durotomy upon deterioration from infarction-related swelling trial: randomized pilot clinical trial. Stroke 2014;45:781-787.

14 Berrrouschot J, Rossler A, Koster J, et al: Mechanical ventilation in patients with hemispheric ischemic stroke. Crit Care Med 2000; 28:2956-2961.

15 Poca MA, Benejam B, Sahuquillo J, et al: Monitoring intracranial pressure in patients with malignant middle cerebral artery infarction: is it useful? J Neurosurg 2010;112:648657.

16 Bardutzky J, Schwab S: Antiedema therapy in ischemic stroke. Stroke 2007;38:3084-3094. 17 Diedler J, Sykora M, Jüttler E, et al: Intensive
care management of acute stroke: general management. Int J Stroke 2009;4:365-378.

18 Jüttler E, Schellinger PD, Aschoff A, et al: Clinical review: therapy for refractory intracranial hypertension in ischaemic stroke. Crit Care 2007;11:231.

19 Diringer MN, Scalfani MT, Zazulia AR, et al: Cerebral hemodynamic and metabolic effects of equi-osmolar doses mannitol and $23.4 \%$ saline in patients with edema following large ischemic stroke. Neurocrit Care 2011;14:11-17.

20 Kamel H, Navi BB, Nakagawa K, et al: Hypertonic saline versus mannitol for the treatment of elevated intracranial pressure: a meta-analysis of randomized clinical trials. Crit Care Med 2011;39:554-559.

21 Hays AN, Lazaridis C, Neyens R, et al: Osmotherapy: use among neurointensivists. Neurocrit Care 2011;14:222-228.

22 Bernard SA, Gray TW, Buist MD, et al: Treatment of comatose survivors of out-of-hospital cardiac arrest with induced hypothermia. N Engl J Med 2002;346:557-563.

23 Van der Worp HB, Macleod MR, Kollmar R; European Stroke Research Network for Hypothermia (EuroHYP): Therapeutic hypothermia for acute ischemic stroke: ready to start large randomized trials? J Cereb Blood Flow Metab 2010;30:1079-1093.

24 Van der Worp HB, Sena ES, Donnan GA, et al: Hypothermia in animal models of acute ischaemic stroke: a systematic review and meta-analysis. Brain 2007;130:3063-3074.

25 Neugebauer H, Kollmar R, Niesen WD, et al.; DEPTH-SOS Study Group; IGNITE Study Group: DEcompressive surgery Plus hypoTHermia for Space-Occupying Stroke (DEPTH-SOS): a protocol of a multicenter randomized controlled clinical trial and a literature review. Int J Stroke 2013;8:383-387.

26 Frank Jl CD, Thisted R, Kordeck C, et al: HeADDIRST trialist: Hemicraniectomy and durotomy upon deterioration from infarction related swelling trial (HeADDIRST): first public presentation of primary study findings. Abstract and scientific session; 55th Annual Meeting of the American Academy of Neurology; March 19th-April 5th 2003.

27 Schwab S, Steiner T, Aschoff A, et al: Early hemicraniectomy in patients with complete middle cerebral artery infarction. Stroke 1998;29:1888-1893.

28 Wang KW, Chang WN, Ho JT, et al: Factors predictive of fatality in massive middle cerebral artery territory infarction and clinical experience of decompressive hemicraniectomy. Eur J Neurol 2006;13:765-771.

29 Maramattom BV, Bahn MM, Wijdicks EF: Which patient fares worse after early deterioration due to swelling from hemispheric stroke? Neurology 2004;63:2142-2145.

30 Malm J, Bergenheim AT, Enblad P, et al: The Swedish Malignant Middle cerebral artery Infarction Study: long-term results from a prospective study of hemicraniectomy combined with standardized neurointensive care. Acta Neurol Scand 2006;113:25-30.

-31 Kilincer C, Asil T, Utku U, et al: Factors affecting the outcome of decompressive craniectomy for large hemispheric infarctions: a prospective cohort study. Acta Neurochir (Wien) 2005; 147:587-594.

32 Robertson SC, Lennarson P, Hasan DM, et al: Clinical course and surgical management of massive cerebral infarction. Neurosurgery 2004;55:55-62. 
33 Foerch C, Lang JM, Krause J, et al: Functional impairment, disability, and quality of life outcome after decompressive hemicraniectomy in malignant middle cerebral artery infarction. J Neurosurg 2004;101:248-254.

34 Chen CC, Cho DY, Tsai SC: Outcome of and prognostic factors for decompressive hemicraniectomy in malignant middle cerebral artery infarction. J Clin Neurosci 2007;14:317 321.

-35 Skoglund TS, Eriksson-Ritzén C, Sörbo A, et al: Health status and life satisfaction after decompressive craniectomy for malignant middle cerebral artery infarction. Acta Neurol Scand 2008;117:305-310.

36 Chung J, Bang OY, Lim YC, et al: Newly suggested surgical method of decompressive craniectomy for patients with middle cerebral artery infarction. Neurologist 2011;17:11-15.

-37 Cho DY, Chen TC, Lee HC: Ultra-early decompressive craniectomy for malignant middle cerebral artery infarction. Surg Neurol 2003;60:227-233.

- 38 Wagner S, Schnippering H, Aschoff A, et al: Suboptimum hemicraniectomy as a cause of additional cerebral lesions in patients with malignant infarction of the middle cerebral artery. J Neurosurg 2001;94:693-696.

-39 Holtkamp M, Buchheim K, Unterberg A, et al: Hemicraniectomy in elderly patients with space occupying media infarction: improved survival but poor functional outcome. I Neurol Neurosurg Psychiatry 2001;70:226-228.

40 Yang XF, Yao Y, Hu WW, et al: Is decompressive craniectomy for malignant middle cerebral artery infarction of any worth? J Zhejiang Univ Sci B 2005;6:644-649.

41 Harscher S, Reichart R, Terborg C, et al: Outcome after decompressive craniectomy in patients with severe ischemic stroke. Acta Neurochir (Wien) 2006;148:31-37.

$\checkmark 42$ Walz B, Zimmermann C, Böttger S, et al: Prognosis of patients after hemicraniectomy in malignant middle cerebral artery infarction. J Neurol 2002;249:1183-1190.

43 Curry WT Jr, Sethi MK, Ogilvy CS, et al: Factors associated with outcome after hemicraniectomy for large middle cerebral artery territory infarction. Neurosurgery 2005;56:681692.

44 Foerch C, Lang JM, Krause J, et al: Functional impairment, disability, and quality of life outcome after decompressive hemicraniectomy in malignant middle cerebral artery infarction. J Neurosurg 2004;101:248-254.

$\checkmark 45$ Park J, Kim E, Kim GJ, et al: External decompressive craniectomy including resection of temporal muscle and fascia in malignant hemispheric infarction. J Neurosurg 2009; 110:101-105.

46 Greenwood J Jr: Acute brain infarctions with high intracranial pressure: surgical indications. Johns Hopkins Med J 1968;122:254260.

47 Ivamoto HS, Numoto M, Donaghy RM: Surgical decompression for cerebral and cerebellar infarcts. Stroke 1974;5:365-370.
48 Güresir E, Vatter H, Schuss P, et al: Rapid closure technique in decompressive craniectomy. J Neurosurg 2011;114:954-960.

49 Lee CH, Cho DS, Jin SC, et al: Usefulness of silicone elastomer sheets as another option of adhesion preventive material during craniectomies. Clin Neurol Neurosurg 2007;109: 667-671.

50 Bulters D, Belli A: Placement of silicone sheeting at decompressive craniectomy to prevent adhesions at cranioplasty. Br J Neurosurg 2010;24:75-76

51 Sobani ZA, Shamim MS, Zafar SN, et al: Cranioplasty after decompressive craniectomy: an institutional audit and analysis of factors related to complications. Surg Neurol In 2011;2:123.

52 Gooch MR, Gin GE, Kenning TJ, et al: Complications of cranioplasty following decompressive craniectomy: analysis of 62 cases. Neurosurg Focus 2009;26:E9.

53 Wachter D, Reineke K, Behm T, et al: Cranioplasty after decompressive hemicraniectomy: underestimated surgery-associated complications? Clin Neurol Neurosurg 2013;115: 1293-1297.

54 Yadla S, Campbell PG, Chitale R, et al: Effect of early surgery, material, and method of flap preservation on cranioplasty infections: a systematic review. Neurosurgery 2011;68:11241130 .

55 Archavlis E, Carvi Y, Nievas M: The impact of timing of cranioplasty in patients with large cranial defects after decompressive hemicraniectomy. Acta Neurochir (Wien) 2012;154: 1055-1062.

56 Piedra MP, Ragel BT, Dogan A, et al: Timing of cranioplasty after decompressive craniectomy for ischemic or hemorrhagic stroke. J Neurosurg 2013;118:109-114.

57 Walcott BP, Kwon CS, Sheth SA, et al: Predictors of cranioplasty complications in stroke and trauma patients. J Neurosurg 2013;118: 757-762.

58 Uhl E, Kreth FW, Elias B, et al: Outcome and prognostic factors of hemicraniectomy for space occupying cerebral infarction. J Neurol Neurosurg Psychiatry 2004;75:270274.

59 Rabinstein AA, Mueller-Kronast N, Maramattom BV, et al: Factors predicting prognosis after decompressive hemicraniectomy for hemispheric infarction. Neurology 2006;67: 891-893.

60 Arac A, Blanchard V, Lee M, et al: Assessment of outcome following decompressive craniectomy for malignant middle cerebral artery infarction in patients older than 60 years of age. Neurosurg Focus 2009;26:E3.

61 Jüttler E, Bösel J, Amiri H, et al; DESTINY II Study Group: DESTINY II: DEcompressive Surgery for the Treatment of malignant INfarction of the middle cerebral arterY II. Int J Stroke 2011;6:79-86.

62 Klein A, Kuehner C, Schwarz S: Attitudes in the general population towards hemi-craniectomy for middle cerebral artery (MCA) in- farction. A population-based survey. Neurocrit Care 2012;16:456-461.

63 Yao Y, Liu W, Yang X, et al: Is decompressive craniectomy for malignant middle cerebral artery territory infarction of any benefit for elderly patients? Surg Neurol 2005;64:165169

64 Weil AG, Rahme R, Moumdjian R, et al: Quality of life following hemicraniectomy for malignant MCA territory infarction. Can J Neurol Sci 2011;38:434-438.

65 Koh MS, Goh KY, Tung MY, et al: Is decompressive craniectomy for acute cerebral infarction of any benefit? Surg Neurol 2000;53: 225-230.

66 Kiphuth IC, Köhrmann M, Lichy C, et al: Hemicraniectomy for malignant middle cerebral artery infarction: retrospective consent to decompressive surgery depends on functional long-term outcome. Neurocrit Care 2010;13: 380-384.

67 Kastrau F, Wolter M, Huber W: Recovery from aphasia after hemicraniectomy for infarction of the speech-dominant hemisphere. Stroke 2005;36:825-829.

68 Berthier ML, Pulvermuller F: Neuroscience insights improve neurorehabilitation of poststroke aphasia. Nat Rev Neurol 2011;7:86-97.

69 Kertesz A, McCabe P: Recovery patterns and prognosis in aphasia. Brain 1977;100:1-18.

70 Robey RR: A meta-analysis of clinical outcomes in the treatment of aphasia. J Speech Lang Hear Res 1998;41:172-187.

71 Hart J: Post-stroke recovery. Alternative and Complimentary Therapies 2010;16:277-280.

-72 Hamilton RH, Chrysikou EG, Coslett B Mechanisms of aphasia recovery after stroke and the role of noninvasive brain stimulation. Brain Lang 2011;118:40-50.

-73 Szaflarski JP, Allendorfer JB, Banks C, et al: Recovered vs. not-recovered from post-stroke aphasia: the contributions from the dominant and non-dominant hemispheres. Restor Neurol Neurosci 2013;31:347-360.

74 Allendorfer JB, Kissela BM, Holland SK: Different patterns of language activation in poststroke aphasia are detected by overt and covert versions of the verb generation fMRI task. Med Sci Monit 2012;18:CR135-CR137.

75 Sahuquillo J, Rădoi A, Benejam B: Brain activation during speech perception in a patient with a massive left hemisphere infarction. Brain Inj 2013;27:1470-1474.

76 Kauhanen ML, Korpelainen JT, Hiltunen P, et al: Aphasia, depression, and non-verbal cognitive impairment in ischaemic stroke. Cerebrovasc Dis 2000;10:455-461.

77 Benejam B, Sahuquillo J, Poca MA, et al: Quality of life and neurobehavioral changes in survivors of malignant middle cerebral artery infarction. J Neurol 2009;256:11261133.

78 Pillai A, Menon SK, Kumar S, et al: Decompressive hemicraniectomy in malignant middle cerebral artery infarction: an analysis of long-term outcome and factors in patient selection. J Neurosurg 2007;106:59-65. 
79 Fischer U, Taussky P, Gralla J, et al: Decompressive craniectomy after intra-arterial thrombolysis: safety and outcome. J Neurol Neurosurg Psychiatry 2011;82:885-887.

80 Leonhardt G, Wilhelm H, Doerfler A, et al: Clinical outcome and neuropsychological deficits after right decompressive hemicraniectomy in MCA infarction. J Neurol 2002; 249:1433-1440.

81 Honeybul S, Ho KM: Long-term complications of decompressive craniectomy for head injury. J Neurotrauma 2011;28:929-935.

82 Durga P, Meena AK, Panigrahi MK, et al: Serious cardiac adverse events after decompressive craniectomy for malignant cerebral infarction. J Neurosurg Anesthesiol 2011;23: 236-240.
83 Akins PT, Guppy KH: Sinking skin flaps, paradoxical herniation, and external brain tamponade: a review of decompressive craniectomy management. Neurocrit Care 2008;9: 269-276.

84 Sarov M, Guichard JP, Chibarro S, et al: Sinking skin flap syndrome and paradoxical herniation after hemicraniectomy for malignant hemispheric infarction. Stroke 2010;41:560562.

85 Gadde J, Dross P, Spina M: Syndrome of the trephined (sinking skin flap syndrome) with and without paradoxical herniation: a series of case reports and review. Del Med J 2012;84: 213-218.

86 Nalbach SV, Ropper AE, Dunn IF, et al: Craniectomy-associated Progressive Extra-Axial Collections with Treated Hydrocephalus (CAPECTH): redefining a common complication of decompressive craniectomy. J Clin Neurosci 2012;19:1222-1227.

87 Lee MH, Yang JT, Weng HH, et al: Hydrocephalus following decompressive craniectomy for malignant middle cerebral artery infarction. Clin Neurol Neurosurg 2012;114: 555-559.
88 Takeuchi S, Takasato Y, Masaoka H, et al: Hydrocephalus following decompressive craniectomy for ischemic stroke. Acta Neurochir Suppl 2013;118:289-291.

89 Takeuchi S, Takasato Y, Masaoka H, et al: Hydrocephalus after decompressive craniectomy for hemispheric cerebral infarction. J Clin Neurosci 2013;20:377-382.

90 Rahme R, Weil AG, Sabbagh M, et al: Decompressive craniectomy is not an independent risk factor for communicating hydrocephalus in patients with increased intracranial pressure. Neurosurgery 2010;67:675-678.

91 Rahme R, Zuccarello M, Kleindorfer D, et al Decompressive hemicraniectomy for malignant middle cerebral artery territory infarction: is life worth living? J Neurosurg 2012. 117:749-754. 